\title{
On the necessity of translational cognitive-neurotoxicological research in methamphetamine abuse and addiction
}

\author{
Ann-Kathrin Stock ${ }^{1} \cdot$ Christian Beste $^{1,2,3}$
}

Received: 18 April 2017 / Accepted: 20 April 2017 / Published online: 25 April 2017

(C) Springer-Verlag Berlin Heidelberg 2017

Keywords Addiction - Methamphetamine - Neurotoxicity · Translational

Humans have been consuming stimulants like amphetamine in the form of cathinone (khat) for thousands of years, but since world war two, synthetic amphetamines, especially methamphetamine (METH), have hit many societies in a worldwide surge (Carvalho et al. 2012). Due to its highly addicting properties and the comparatively low cost, the abuse of this psychostimulant has rapidly increased in the last decades and years (Chomchai and Chomchai 2015) so that METH has become one of the most abused substances worldwide (Bernheim et al. 2016). Continued METH consumption does not only have devastating effects on the health and well-being of the addicted individuals, but also affects whole communities and put a considerable strain on healthcare systems (Panenka et al. 2013; Chomchai and Chomchai 2015). In addition, while all forms of METH toxicity are undoubtedly harmful to the affected individual, we think that it is worth to direct special attention to the neurotoxic effects and as the associated neurocognitive impairments (especially deficits in executive functioning and inhibition) increase the risk of poorer health outcomes,

Christian Beste

christian.beste@uniklinikum-dresden.de

1 Cognitive Neurophysiology, Department of Child and Adolescent Psychiatry, Faculty of Medicine, TU Dresden, Schubertstr. 42, 01307 Dresden, Germany

2 Experimental Neurobiology, National Institute of Mental Health, Klecany, Czech Republic

3 Cognitive Neurophysiology, Department of Child and Adolescent Psychiatry, Faculty of Medicine, TU Dresden, Fetscherstrasse 74, 01307 Dresden, Germany high-risk behaviors, treatment non-adherence, and repeated relapses. This ultimately contributes to maintaining continuous drug-seeking behaviors (Cadet and Bisagno 2015), and thus indirectly enhances all of the toxicological effects of METH abuse. METH is often consumed for its effects which, among others, include increased energy levels and decreases in fatigue, increased psychomotor activity and alertness, anorectic effects, increases in sex drive, and euphoria. Yet, irritability, agitation, risky sexual behaviors, (motor) stereotypies, psychosis, seizures, hyperthermia and other systemic effects are also common acute (side) effects of METH consumption (Panenka et al. 2013; Cadet and Bisagno 2015; Chomchai and Chomchai 2015). Those effects are mainly attributed to increases in both central and peripheral monoaminergic signaling, with strong effects in the noradrenergic (NA) and dopaminergic (DA) systems (5-HT) (Schep et al. 2010; Carvalho et al. 2012). Repeated consumption causes striking and long-lasting dysfunctions in monoaminergic signaling which are characterized by decreases in the levels of monoamines and their metabolites, monoamine transporter-binding sites, lower expression and/or activity of enzymes involved in the synthesis and metabolism of brain monoamine neurotransmitters, and neurodegeneration (especially of dopaminergic nerve terminals) (Carvalho et al. 2012; Halpin et al. 2014). Other indirect acute neuronal effects of METH include the release of glutamate in cortical and subcortical regions as well as glia activation (Rocher and Gardier 2001; Carvalho et al. 2012).

While these effects are well known, they have mostly been regarded to affect the consuming individual. In this regard, recent findings in animals by Fujaková-Lipski et al. (2017) have shown transgenerational effects of METH exposure by looking at how neurotoxic effects of METH administered in utero alter various neurotransmitter 
systems in their offspring. The results show that METH exposure decreased basal levels of monoamines and GABA, but increased glutamate levels in all measured brain regions. Acute challenge with METH injection in the METH-exposed group induced a lower increase in monoamine system relative to the increase in the GABAergic and glutamatergic system. The data show that prenatal METH exposure has strong effects on the monoaminergic, GABAergic and glutamatergic system even when exposure to methamphetamine was limited to the prenatal phase. Toxicological effects of METH have, therefore, longer lasting effects as currently considered and affect the dopaminergic system. This is of tremendous importance, because the dopaminergic system plays a major role in several cognitive processes that are relevant for everyday life situations (Diamond 2013). Of note, it has been hypothesized that METH-induced changes in dopaminergic signaling involve a change in set point for drug reward that may represent an allostatic state contributing to vulnerability to relapse and re-entry into the addiction cycle (Koob 2000). It is, therefore, likely that the already observed dysfunction of prefrontal and limbic brain areas as well as fronto-striatal loops, which has repeatedly been reported in regular METH consumers (Chang et al. 2007; Salo et al. 2011; Bisagno et al. 2016; Okada et al. 2016), is also evident in their offspring. Given that cognitive functions mediated via the prefrontal cortex are highly predictive for school readiness, school success, job success, public safety, physical and mental health (Diamond 2013), it is likely that transgenerational effects are devastating for the upcoming generations and lead to strong societal problems. Aside malfunctioning of different cognitive functions, it is likely that such transgenerational effects may induce a high likelihood that the offspring of abusing mothers will also become affected by some sort of drug addiction. This induces a vicious cycle that is hard to burst. Such processes have however not been focused in research on the effects METH consumption and reflect an upcoming field of cognitive-neurotoxicological research. This will be important to determine the severity of possible adverse effects.

In this regard, it is important to consider that the vast majority of research on the effect of METH is performed either using animals or by evaluating the effects of METH abuse in addicted humans. Animal studies allow for pharmacologically controlled substance administration and several histologic procedures that must not be conducted in humans due to ethical reasons. Yet, most animal studies do not allow for a direct inference on the processes in humans due to differences in pharmacokinetics, cellular functioning, dose differences, and behavioral differences. Human subjects, on the other hand, allow for direct inferences about the effects of METH. Yet, many factors can hardly be controlled in human samples and the results are quite often confounded by comorbidities or polysubstance abuse. Given that both lines of research have advantages and drawbacks, it should, therefore, prove beneficial to combine research in animals and humans by taking converging approaches. Doing so would allow to examine the same (or at least corresponding) neurobiochemical, neurophysiological/electrophysiological, behavioral, or cognitive processes in both humans and animal models to overcome some of the limitations of each group and provide a more causal, mechanistic insight into the effects of METH toxicity and its consequences. This necessitates closely coordinated collaborations, which, despite the anticipated effort of consultation and coordination, may provide fruitful new insights and help further integrate the findings made in both fields (van Thriel et al. 2012, 2017). As part of these efforts particular attention must be paid to examine the neurobiological and neurophysiological mechanisms underlying possible dysfunctions in cognitive processes relevant to the above-mentioned fields of school readiness, school success, job success, public safety, physical and mental health (Diamond 2013). In this way, basic cognitive-neurotoxicological research with a clear translational focus on research in animals and humans will have long-lasting beneficial effects on policy and society.

Acknowledgements This work was partly supported by a Grant from the Deutsche Forschungsgemeinschaft (DFG) SFB 940 project B8.

\section{References}

Bernheim A, See RE, Reichel CM (2016) Chronic methamphetamine self-administration disrupts cortical control of cognition. Neurosci Biobehav Rev 69:36-48. doi:10.1016/j. neubiorev.2016.07.020

Bisagno V, González B, Urbano FJ (2016) Cognitive enhancers versus addictive psychostimulants: the good and bad side of dopamine on prefrontal cortical circuits. Pharmacol Res 109:108-118. doi:10.1016/j.phrs.2016.01.013

Cadet JL, Bisagno V (2015) Neuropsychological consequences of chronic drug use: relevance to treatment approaches. Front Psychiatry 6:189. doi:10.3389/fpsyt.2015.00189

Carvalho M, Carmo H, Costa VM et al (2012) Toxicity of amphetamines: an update. Arch Toxicol 86:1167-1231. doi:10.1007/ s00204-012-0815-5

Chang L, Alicata D, Ernst T, Volkow N (2007) Structural and metabolic brain changes in the striatum associated with methamphetamine abuse. Addict Abingdon Engl 102(Suppl 1):16-32. doi:10.1111/j.1360-0443.2006.01782.x

Chomchai C, Chomchai S (2015) Global patterns of methamphetamine use. Curr Opin Psychiatry 28:269-274. doi:10.1097/ YCO.0000000000000168

Diamond A (2013) Executive functions. Annu Rev Psychol 64:135168. doi:10.1146/annurev-psych-113011-143750

Fujaková-Lipski M, Kaping D, Sirova J et al (2017) Transgenerational neurobiochemical modulation of methamphetamine in the adult brain of the Wistar rat. Arch Toxicol (in press) 
Halpin LE, Collins SA, Yamamoto BK (2014) Neurotoxicity of methamphetamine and 3,4-methylenedioxymethamphetamine. Life Sci 97:37-44. doi:10.1016/j.lfs.2013.07.014

Koob GF (2000) Neurobiology of addiction. Toward the development of new therapies. Ann N Y Acad Sci 909:170-185

Okada N, Takahashi K, Nishimura Y et al (2016) Characterizing prefrontal cortical activity during inhibition task in methamphetamine-associated psychosis versus schizophrenia: a multi-channel near-infrared spectroscopy study. Addict Biol 21:489-503. doi:10.1111/adb.12224

Panenka WJ, Procyshyn RM, Lecomte T et al (2013) Methamphetamine use: a comprehensive review of molecular, preclinical and clinical findings. Drug Alcohol Depend 129:167-179. doi:10.1016/j.drugalcdep.2012.11.016

Rocher C, Gardier AM (2001) Effects of repeated systemic administration of d-Fenfluramine on serotonin and glutamate release in rat ventral hippocampus: comparison with methamphetamine using in vivo microdialysis. Naunyn Schmiedebergs Arch Pharmacol 363:422-428

Salo R, Ravizza S, Fassbender C (2011) Overlapping cognitive patterns in schizophrenia and methamphetamine dependence. Cogn Behav Neurol Off J Soc Behav Cogn Neurol 24:187-193. doi:10.1097/WNN.0b013e31823fc1d0

Schep LJ, Slaughter RJ, Beasley DMG (2010) The clinical toxicology of metamfetamine. Clin Toxicol Phila Pa 48:675-694. doi:10.31 09/15563650.2010.516752

van Thriel C, Westerink RHS, Beste C et al (2012) Translating neurobehavioural endpoints of developmental neurotoxicity tests into in vitro assays and readouts. Neurotoxicology 33:911-924. doi:10.1016/j.neuro.2011.10.002

van Thriel C, Quetscher C, Pesch B et al (2017) Are multitasking abilities impaired in welders exposed to manganese? Translating cognitive neuroscience to neurotoxicology. Arch Toxicol. doi:10.1007/s00204-017-1932-y 